\title{
Concurrent staphylococcal and herpes simplex infections - diagnostic pitfalls. A case report
}

\author{
Wojciech Walas ${ }^{1}$, Zenon P. Halaba² \\ 'Paediatric and Neonatal Intensive Care Unit, University Hospital in Opole, Opole, Poland \\ ${ }^{2}$ Institute of Medicine, University of Opole, Opole, Poland
}

\section{ABSTRACT}

We describe the case of a six-year-old boy with concurrent staphylococcal septicaemia, herpes simplex virus encephalitis (HSVE), and haemorrhagic erosive oesophagitis. Due to guiding clinical and lab symptoms that suggested a diagnosis of staphylococcal sepsis with central nervous system (CNS) involvement, acyclovir treatment was discontinued after three days, but the lack of progress in neurological status, further lab tests, and CT and MRI imaging led the authors to consider possible HSV infection, and this diagnosis was confirmed by PCR test. Even though the treatment for HSV was applied again, the long gap in acyclovir medication contributed to a severe neurological deficit and an adverse outcome.

Our case strongly suggests the need to consider HSV aetiology in making a differential diagnosis where there is an atypical course of a disease, even though clinical symptoms and lab results dictate another aetiology and continuing acyclovir supply until HSV aetiology is ruled out.

KEY WORDS:

herpes simplex virus encephalitis, staphylococcal septicaemia, neurological sequelae, and children.

\section{INTRODUCTION}

Herpes simplex viruses (HSV) are among the most common widespread causative agents of human viral infections. HSV-1 is associated primarily with orolabial ulceration, while HSV-2 is one of the most common causes of genital disease. However, recent changes in HSV epidemiology have shown an increase in genital and neonatal herpes, particularly caused by HSV-1. Herpes simplex virus is a common cause of encephalitis, with an incidence of 1-4 cases per million inhabitants each year. It is a potentially devastating disease with significant rates of mortality and co-morbidities $[1,2]$. Recent research suggests that an inborn error of interferon-mediated immunity may predispose the HSV-1 infected individual to developing HSVE [3-5].

\section{CASE REPORT}

An unconscious six-year-old boy was admitted to the paediatric intensive care unit due to upper gastrointestinal bleeding. On admission, the laboratory data revealed severe anaemia (haemoglobin $-6.4 \mathrm{~g} / \mathrm{dl}$, haematocrit $-20 \%$, RBC -2.1 million cells/mcl); leukocytes, procalcitonin, and C-reactive protein were elevated (WBC 24,300 cells/mcl, CRP - 199 mg/dl, PCT 42 ng/ml). The leukogram showed abnormally elevated levels of neutrophils (e.g., 83\%) and low levels of lymphocytes (e.g., 7\%). The platelet count scarcely exceeded the upper limit of the normal range $\left(430 \times 10^{9} / 1\right)$, and in the coagulogram, only the D-dimer level was slightly elevated $(1967 \mu \mathrm{g} / \mathrm{l})$. Endoscopic examination revealed haemorrhagic erosive oesophagitis in its lower part. The child required intuba-

\section{ADDRESS FOR CORRESPONDENCE:}

Zenon P. Halaba, Institute of Medicine, University of Opole, 48 Oleska St., 45-052 Opole, Poland,

e-mail: zhalaba@uni.opole.pl 
tion and ventilation. Fluids, catecholamines, inotropes, vasopressin, proton pump inhibitors, vitamin $\mathrm{K}$, red cell concentrates, and fresh frozen plasma were administered. Empirical antibiotic therapy with ampicillin and cefotaxime was initiated. He was also given acyclovir as a precautionary treatment for encephalitis. Due to the persistent bleeding from the lower end of the oesophagus, despite the lack of coagulation abnormalities, a Sengstaken-Blakemore tube was inserted and placed under tension. Successful haemostasis was subsequently achieved, and the patient remained stable. Once his medical condition had stabilised, he had a lumbar puncture, and cerebrospinal findings were characteristic of acute communityacquired bacterial meningitis (Pandy's test: $(+++)$, NonneApelt test: $(++)$, glucose $-9 \mathrm{mg} / \mathrm{dl}$, proteins $-314 \mathrm{mg} / \mathrm{dl}$, WWBC - 1250 cells/ $\mu$ l, polymorphonucleocytes - 95\%, lymphocytes $-4 \%$, monocytes - 1\%). Methicillin-resistant Staphylococcus warneri was identified from the patient's blood culture, and cerebrospinal fluid (CSF) culture was negative. Antibiotic therapy was guided by sensitivities and, based on the result of an antibiogram, the patient was started on vancomycin. Furthermore, despite negative CSF bacterial culture, bearing in mind the cerebrospinal fluid findings distinctive of bacterial meningitis, antibiotic coverage was extended to meropenem. But unfortunately, acyclovir therapy was discontinued. Although his cardiovascular and respiratory status, as well as lab tests, had improved, his neurological status remained severe. He was unconscious, despite the discontinuation of sedatives. On day 10 after admission, a contrast MRI scan of the head demonstrated the abnormalities specific to encephalitis in the course of HSV infection. At the same time a positive HSV-1-specific polymerase chain reaction (PCR) test result in CSF confirmed herpes simplex type 1 infection. Hence, he was medicated with acyclovir again. Although the rehabilitation was continued and his respiratory and haemodynamic status were good and stable, he still remained unconscious, presenting flaccid paralysis of the lower limbs. Considering genetically determined susceptibility to herpes simplex encephalitis, molecular investigations were projected, but the parents did not consent to them. The patient was discharged home in a persistent vegetative state requiring 24-hour care.

\section{DISCUSSION}

Childhood and adult HSV encephalitis are usually due to HSV-1 (90\%), with the rest due to HSV-2 [6]. Although CSF testing is an essential component of diagnostic workup and can show elevated lymphocytes and protein levels, characteristic findings from neuroimaging studies by CT or MRI contribute significantly to the diagnosis of herpes simplex virus encephalitis (HSVE) [7]. HSV-specific polymerase chain reaction in the cerebrospinal fluid proved to be a gold standard of high sensitivity and specificity [8]. Early antiviral treatment seems to be a strong predictor of mortality and adverse outcome; thus, early empirical antiviral treatment is strongly recommended before the aetiology is proven, and acyclovir remains the treatment of choice. In terms of treatment, data clearly indicate that an elapsed time of longer than three days between the onset of symptoms and initiation of effective antiviral therapy correlates directly with a less-favourable neurological outcome. Thus, the initiation of high-dose acyclovir therapy as early as possible in the course of illness provides the best chance for a patient to survive with minimal neurological damage [9]. Herpes simplex oesophagitis is an uncommon condition, which occurs mainly in immunosuppressed patients, but has rarely been reported in immunocompetent individuals [10]. In our patient, staphylococcal septicaemia in association with herpes simplex encephalitis, which is a rare scenario, led to a diagnostic delay with regard to the second disease because the assumption was that staphylococcal septicaemia was the cause of all the major presenting features. It is important to remain vigilant in patients, even when the primary diagnosis is acquired. It seems to be more likely that in our patient the underlying condition was HSV infection in the form of encephalitis and erosive oesophagitis and viraemia. Supposed staphylococcal superinfection seems to be a result of bacterial translocation through the affected wall of the oesophagus. Thus, guiding clinical and lab symptoms suggested a diagnosis of staphylococcal sepsis with central nervous system involvement. Notwithstanding the fact that $S$. warneri was the putative causative agent of the infection, doubts should have remained with regard to the validity of species identification and the association with a process of infection. Although on the first day the patient had received empirical antibiotic therapy together with acyclovir, on the third day acyclovir was discontinued because the blood culture result suggested S. warneri as the culprit. Moreover, other blood tests and CSF analysis were typical of bacterial infection, but the lack of progress in neurological status and further lab tests led us to think about possible HSV infection, and this diagnosis was confirmed by PCR test. Even though the treatment for HSV was applied again, the long gap in acyclovir medication certainly contributed to a severe neurological deficit and an adverse outcome. It should be emphasised that discontinuance of acyclovir treatment before ruling out HSVE was a false step. To date, we have not found in the literature a case of a child suffering from staphylococcal septicaemia and HSV viraemia, HSVE, and haemorrhagic erosive oesophagitis concurrently.

\section{CONCLUSIONS}

This case strongly suggests the need to consider HSV aetiology in making a differential diagnosis where there is an atypical course of disease. Acyclovir should not be stopped until HSV aetiology is ruled out, even though clinical symptoms and lab results dictate another aetiology. 


\section{DISCLOSURE}

The authors declare no conflict of interest.

\section{REFERENCES}

1. Schmutzhard E. Viral infections of the CNS with special emphasis on herpes simplex infections. J Neurol 2001; 248: 469-477.

2. Whitley RJ, Gnann JW. Viral encephalitis: familiar infections and emerging pathogens. Lancet 2002; 359: 507-513.

3. Gnann JW Jr, Whitley RJ. Herpes simplex encephalitis: an update. Curr Infect Dis Rep 2017; 19: 13.

4. Zhang SY, Abel L, Casanova JL. Mendelian predisposition to herpes simplex encephalitis. Handb Clin Neurol 2013; 112: 1091-1097.

5. Menasria R, Boivin N, Lebel M, et al. Both TRIF and ISP-1 adaptor proteins contribute to the cerebral innate immune response against herpes simplex virus 1 infection. J Virol 2013; 87: 7301-7308.

6. Steiner I, Kennedy PG, Pachner AR. The neurotropic herpes viruses: herpes simplex and varicella-zoster. Lancet Neurol 2007; 6: 1015-1028.

7. Chow FC, Glaser CA, Sheriff $\mathrm{H}$, et al. Use of clinical and neuroimaging characteristics to distinguish temporal lobe herpes simplex encephalitis from its mimics. Clin Infect Dis 2015; 60: 1377-1383.

8. López Roa P, Alonso R, de Egea V, et al. PCR for detection of herpes simplex virus in cerebrospinal fluid: alternative acceptance criteria for diagnostic workup. J Clin Microbiol 2013; 51: 2880-2883.

9. Hsieh WB, Chiu NC, Hu KC, et al. Outcome of herpes simplex encephalitis in children. J Microbiol Immunol Infect 2007; 40: 34-38.

10. Al-Hussaini AA, Fagih MA. Herpes simplex ulcerative esophagitis in healthy children. Saudi J Gastroenterol 2011; 17: 353-356. 
